\title{
Role of dexamethasone in peritonsillar abscess
}

\author{
Jitendra Nagar ${ }^{1 *}$, Vijay Kumar Meena ${ }^{2}$, Shamendra Kumar ${ }^{3}$ \\ ${ }^{1,3}$ Medical Officer, ${ }^{2}$ Professor, ${ }^{1}$ Jhalawar Medical College, Jhalawar, Rajasthan, ${ }^{2}$ Government Medical College, Kota, \\ Rajasthan, ${ }^{3}$ M.B.S. Hospital, Kota, Rajasthan, India \\ *Corresponding Author: Jitendra Nagar \\ Email: shamendra.meena82@gmail.com
}

\begin{abstract}
The most important finding in oral foul smelling is abscess. Peritonsillar abscess it is due to tuberculosis or chronic disease. Incision and drainage is treatment of choice and systematic antibiotics and pain killer, gargle and if itiology is tuberculosis than start ATT.
\end{abstract}

Keywords: ATT, PT, I \& D.

\section{Introduction}

Peritonsillar abscess was first described as early as the 14th century; also known as Quincy, is the collection of pus in peritonsillar space. It is one of the most common presentations to otolaryngologist, with the incidence around 30 case per 1,00000 population. The use of systemic corticosteroids along with antibiotics in peritonsillar abscess can reduce pain and truisms. Resulting in an earlier return to normal diet and activities. Their use is widespread but inconsistent, perhaps due to concerns about the immediate immunosuppressive effect and long- term side effects associated with these drugs. A Peritonsillar abscess is a localized accumulation of pus in the peritonsillar tissues that forms as a result of Suppurative tonsillitis or alternative explanation is that a Peritonsillar abscess is an abscess for median group of salivary glands in the supra tonsillar fossa, known as Weber's glands. The nidus of accumulation is located between the capsule of the palatine tonsils and the constrictor muscles of pharynx.

The anterior and posterior pillar, torus tuberous [superior] and pyriform sinus (inferior) form the boundaries of this potential peritonsillar space. Because this area is composed of loose connective tissue, severe infection may rapidly lead to formation of purulent material. Progressive inflammation and suppuration may extend to directly involve the soft palate, the lateral wall of the pharynx, and, occasionally, the base of the tongue.

\section{Clinical presentation}

Peritonsillar abscess typically present with history of acute pharyngitis accompanied by tonsillitis and worsening unilateral pharyngeal discomfort. Patients also may experience malaise; fatigue, headaches. They often present with a fever and asymmetric throat fullness. Associated halitosis, odynophagia, dysphagia and a 'hot potato sounding' voice occurs. Many patient present with ipsilateral referred otalgia with swallowing, Trismus [limitation in the ability to open the oral cavity] of varying severity is present in all cases, reflecting lateral pharyngeal wall and pterygoid musculature inflammation. Because of lymphadenopathy and cervical muscle inflammation, patients often experience neck pain and even limitation in neck mobility. As the degree of inflammation and infection proceeds, symptoms include progression in the floor of mouth, Parapharyngeal space and the prevertebral space Extension in the floor of mouth is worrisome because of airway obstruction. The presentation may range from acute tonsillitis with unilateral pharyngeal asymmetry to dehydration and sepsis most patient have severe pain Examination of the oral cavity reveals marked erythema asymmetry of the soft palate, tonsillar exudation and contra lateral displacement of the uvula .soft palate is erythematous and edematous is displaced anteriorly. Unilateral swelling of peritonsillar area and soft palate with anterior displacement of ipsilateral tonsil and persistent enlargement, Uvular deviation; trismus and 
inferior displacement of the superior pole of the affected side. Degree of trismus depends on extent of lateral pharyngeal space inflammation. Tender ipsilateral cervical lymphadenopathy involving single or multiple nodes. Affected lymph nodes may be quit firm. In present with significant nodal inflammation the patient may experience torticollis and limitation of neck mobility.

\section{Treatment and Management Surgical drainage}

In peritonsillar abscess when incision and drainage/needle aspiration is performed; it lead to immediate improvement of patients symptoms. The aspiration fluid sent for culture and sensitivity and in some case incision and drainage/needle aspiration not necessary. If patient continue to report recurring or chronic sore throats after proper Incision and drainage. A tonsillectomy may be indicated. Intraoral drainage has a high rate of success and low rate of recurrence and morbidity. In situations in which the abscess is located in an area of difficult to access a tonsillectomy may be the only way to drain abscess.

\section{Medical Therapy}

Patients with peritonsillar abscess who are dehydrated require intravenous fluid administration until the inflammation resolve and they are able to resume an adequate oral fluid intake. Antipyretics and analgesic are used to alleviate fever and discomfort. Oral and parenteral analgesics are an integral part of the management and allow the patient to resume oral intake. Pain relief after incision and drainage so significant that the patient is able to resume oral intake with nonnarcotic analgesic. Antibiotic therapy should begin immediately during admission. High dose penicillin IV remains a good choice for empirical treatment of peritonsillar abscess. Alternatively, because of polymicrobial nature of cultured pus, agents that treat co pathogen and resist beta lactamases also have been recommended as a first choice. Cephalexin or another cephalosporin [with or without metronidazole] is likely the best initial option. Alternatives drugs are Cefuroxime or cefpodoxime [with or without metronidazole], clindamycin, Trovafloxacin, Amoxicillin-clavulanate mononucleosis has been ruled out] Oral antibiotics may be prescribed once the patient is able to tolerate oral intake, some studies show that use of IV steroids [Dexamethasone, Methyle-prednisoloneolone].

\section{Etiology $=$}

Any of the microorganisms that cause acute or chronic tonsillitis may be the cause of a Peritonsillar abscess. Most commonly, aerobic and anaerobic gram-positive organisms are identified by means of culture. Cultures of affected patients reveal group A beta hemolytic streptococci as most prevalent. Next most commonly, staphylococci, pneumococci, and Haemophilus organisms are found. Finally, other microorganisms that can be cultured include lactobacilli, filamentous forms such as Actinomyces species, micrococci, Neisseria species, diphtheroids, Bacteroides species, and nonsporulating bacteria. Some evidence indicates that anaerobic bacteria frequently cause these infections.

\section{Complications}

Numerous clinical complications may occur if the diagnosis of a peritonsillar abscess is missed or delayed. The severity of the complications depends on the rapidity of progression of the illness, as well as the characteristics of the affected fascial spaces. Early management and intervention are important. The fascial spaces of the neck are interconnected. Once inflammation exceeds the limits of the peritonsillar space, involvement of the masticator space (with increasing degrees of trismus) occurs. Extension may progress to the submandibular and sublingual spaces within the floor of the mouth (Ludwig angina). At this point, emergency airway control through intubation or tracheotomy is indicated to obviate obstruction from swelling of the base of the tongue. In severe cases, death may occur. In patients treated with I\&D, evaluating the patency of the opening is important. Premature closure before the cavity has become obliterated is possible, leading to reaccumulation of pus. This may warrant a second I\&D procedure or a tonsillectomy. Limiting the drainage incision to the mucosa of the soft palate and using blunt dissection within the cavity are important for preventing serious bleeding. The terminal branches of the external carotid 
artery lie on the posterior aspect of the tonsillar fossa and can be injured easily, particularly in children, in whom they are relatively superficial. Bleeding is a potential complication if branches of the external carotid artery are injured or if the external carotid artery itself is injured. The bleeding may occur intra operatively or in the early postoperative period. Intraoprative hemorrhage is an emergency and results from direct injury to the external carotid artery or terminal branches thereof. Once the patient is stabilized thermodynamically, the tonsillar fossa is reevaluated. The ipsilateral neck also should be prepared and draped in a sterile fashion to provide access to the proximal external carotid artery. If the hemorrhage is controlled intraoral, the patient's continued stabilization is pursued. If the bleeding appears to be too brisk, and it is not controlled by careful intraoral source identification, an ipsilateral cervicotomy is performed, as follows. The sternocleidomastoid is retracted laterally, and the carotid sheath contents are identified. The internal jugular vein, the vagus nerve, and the carotid arteries (common, external, and internal) are identified. A vascular loop is applied around the external carotid artery to assess temporization of bleeding. The external carotid artery is dissected superiorly, with careful attention to preserving the external laryngeal, the ansa hypoglossi, and the hypoglossal nerves. Ligation of the external carotid artery may prove necessary. The general approach to postoperative hemorrhage similarly is directed to the identification of the source of bleeding. The patient is brought to the operating room, and the same procedure as described above is followed. Other well reported complications due to continued progression of the infection may lead to a parapharyngeal abscess, descending necrotizing mediastinitis, Lemierre syndrome, and internal carotid artery pseudoaneurysm.

\section{Review of Literature}

Glucocorticosteroids have some benefits in outcome in peritonsillar abscess that is supported by some studies that is in favor of it. Discuss one by one.

A controlled trial with concealed allocation was conducted at two Canadian EDs. Following written informed consent, eligible patients received 48 hours of intravenous clindamycin and a single dose of the study drug (dexamethasone [DEX], intravenously [IV]). Follow-up occurred at 24 hours, 48 hours, and 7 days. The primary outcome was pain; other outcomes were side effects and return to normal activities/diet. A total of 182 patients were screened for eligibility; 41 patients were enrolled (21 DEX; 20 PLAC). At 24 hours, those receiving DEX reported lower pain scores (1.4 vs. 5.1; $\mathrm{P}=.009$ ); however, these differences disappeared by 48 hours $(\mathrm{P}=.22)$ and 7 days $(\mathrm{P}=.4)$. At 24 hours, more patients receiving DEX returned to normal activities (33\% vs. $11 \%$ ) and dietary intake (38\% vs 25\%); however, these differences were not significant and disappeared by 48 hours and 7 days. Side effects were rare and did not differ between groups $(\mathrm{P}>$.05).Combined with PTA drainage and IV antibiotics, $10 \mathrm{mg}$ IV DEX resulted in less pain at 24 hours when compared to PLAC, without any serious side effects. This effect is short-lived, and further research is required on factors associated with PTA treatment success.

\section{Source of funding}

None.

\section{Conflict of interest}

None.

\section{References}

1. Herzon FS. Harris P. Mosher Award thesis. Peritonsillar abscess: incidence, current management practices, and a proposal for treatment guidelines. Laryngoscope 1995;105 (supple 74):1-17.

2. Millar KR, Johnson DW, Drummond D, Kellner JD. Suspected peritonsillar abscess in children. Pediatr Emerg Care 2007;23:431-8.

3. Ozbek C, Aygenc E, Tuna EU, Selcuk A, Ozdem C. Use of steroids in the treatment of peritonsillar abscess. $J$ Laryngol Otol 2004;118:439-42.

4. Chau JKM, Seikaly HR, Harris JR, Villa-Roel C, Brick C, Rowe BH. Corticosteroids in peritonsillar abscess treatment: a blinded placebo-controlled clinical trial. Laryngoscope 2014;124:97-103. doi: 10.1002/lary.24283.

5. Hayward G, Thompson MJ, Perera R, Glasziou PP, Del Mar CB, Heneghan CJ. Corticosteroids as standalone or add-on treatment for sore throat. Cochrane Database Syst Rev 2012;10:CD008268. doi:

10.1002/14651858.CD008268.pub2 [Am Fam Physician. 2014] 
6. Sculpher M, Drummond M, O’Brien B. Effectiveness, efficiency, and NICE. BMJ 2001;322:943-4.

7. Russell LB. Opportunity costs in modern medicine. Health Affairs 1992;11:162.

8. Gold MR, Siegel JR, Russell LB. Cost effectiveness in health and medicine. New York: Oxford University Press, 1996.

9. Palmer S, Rafferty J. Economic notes: opportunity cost. BMJ 1999;318:1551-2.

10. Hall SF. Peritonsillar abscess: the treatment options. J Otol 1990;19:2269.

How to cite this article: Nagar J, Meena VK, Shamendra. Role of dexamethasone in peritonsillar abscess. Indian J Anat Surg Head Neck Brain 2019;5(3):82-5. 